\title{
Anti-apoptotic effect of hyperglycemia can allow survival of potentially autoreactive T cells
}

\author{
P Ramakrishnan ${ }^{1,3}$, DA Kahn ${ }^{1,2,3}$ and D Baltimore ${ }^{*, 1}$
}

Thymocyte development is a tightly controlled multi-step process involving selective elimination of self-reactive and nonfunctional T cells by apoptosis. This developmental process depends on signaling by Notch, IL-7 and active glucose metabolism. In this study, we explored the requirement of glucose for thymocyte survival and found that in addition to metabolic regulation, glucose leads to the expression of anti-apoptotic genes. Under hyperglycemic conditions, both mouse and human thymocytes demonstrate enhanced survival. We show that glucose-induced anti-apoptotic genes are dependent on NF- $\kappa \mathrm{B}$ p65 because high glucose is unable to attenuate normal ongoing apoptosis of thymocytes isolated from p65 knockout mice. Furthermore, we demonstrate that in vivo hyperglycemia decreases apoptosis of thymocytes allowing for survival of potentially self-reactive thymocytes. These results imply that hyperglycemic conditions could contribute to the development of autoimmunity through dysregulated thymic selection.

Cell Death and Differentiation (2011) 18, 690-699; doi:10.1038/cdd.2010.163; published online 17 December 2010

Diabetes has reached epidemic proportions and as a result, the complications of un-opposed hyperglycemia are growing in magnitude. Identification of the molecular mechanisms underlying the causes of diabetic complications is an area of active research. Unfortunately, diabetes is increasingly affecting the lives of children and fetuses. ${ }^{1}$ Gaining a clear understanding of the developmental impacts of diabetes is crucial and relatively unexplored.

T-cell development is a highly orchestrated process harmonized with extensive apoptosis to eliminate selfreactive and non-functional T cells. More than $95 \%$ of the thymocytes generated die within the thymus and only $2-4 \%$ leave the thymus as mature $\mathrm{T}$ cells. Thymocytes generally progress through three different maturational stages that can be identified based on the surface expression of T-cell co-receptors CD4 and CD8: $\mathrm{CD}^{-} \mathrm{CD}^{-}$double negative (DN), $\mathrm{CD}^{+}{ }^{+} \mathrm{CD} 8^{+}$double positive (DP), then either $\mathrm{CD}^{+}$or $\mathrm{CD}^{+}$single positive (SP). Several checkpoints including rearrangement of T-cell antigen receptors, signaling by IL-7 and the developmental ligand Notch, as well as T-cell bioenergetics has been suggested to decide T-cell fate..$^{2,3}$ Apoptosis has a critical role at each stage to eliminate undesirable $\mathrm{T}$ cells. At the DN and DP stage, thymocytes without a functional T-cell receptor (TCR) undergo apoptosis. Surviving thymocytes that become SP, migrate to the thymic medulla in which if they recognize peptide (presumably self) presented by $\mathrm{MHC}$, they undergo apoptosis. Through this stringent process collectively known as central tolerance, both non-functional $T$ cells and $T$ cells that recognize self-antigens are eliminated. 4

Developing thymocytes depend on glucose for energy production as well as proliferation. ${ }^{5}$ Many studies suggest a key role for glucose and glucose metabolism in shaping the thymocyte apoptotic profile. ${ }^{6}$ IL-7 and Notch signaling, both crucial for thymocyte development, have been shown to be essential for maintaining thymocyte glucose metabolism and cell viability during thymic selection. ${ }^{2,7}$ Glucose has been suggested to exert anti-apoptotic effects in non-thymocytes by several mechanisms. For instance, through enhanced cell viability by increasing cellular ATP levels through the glycolytic pathway or by increasing NADPH, through the pentose phosphate pathway. ${ }^{8}$ Both pathways have been suggested to inhibit caspase activation in other cell types. ${ }^{9,10}$ Glucose has also been shown to induce direct binding of hexokinase to the voltage-dependent anion channel (VDAC) in mitochondria blocking cytochrome $c$ release and prevent apoptosis. ${ }^{11}$ However, little is known in thymocytes about the molecular mechanisms behind glucose metabolism-mediated decrease in apoptosis. Less is known about whether an anti-apoptotic machinery exists in thymocytes that might be inducible by glucose.

$\mathrm{NF}-\kappa \mathrm{B}$ is one of the major determinants of anti-apoptotic effects in many cell types. NF- $\kappa$ B represents a group of evolutionary conserved and structurally related eukaryotic transcription factors comprising five members: p65 (RelA), RelB, c-Rel, NF- $\kappa$ B1 (which occurs both as a precursor, p105,

${ }^{1}$ Division of Biology, California Institute of Technology, 1200 California Boulevard, Pasadena, CA 91125, USA and ${ }^{2}$ Division of Maternal-Fetal Medicine, Department of OB/GYN, David Geffen School of Medicine at UCLA, BOX 951740, 22-168 CHS, Los Angeles, CA 90095-1740, USA

${ }^{*}$ Corresponding author: D Baltimore, Division of Biology, California Institute of Technology, Mail Code 147-75, 1200 California Boulevard, Pasadena, CA 91125, USA. Tel: + 626395 3581; Fax: + 626585 9495; E-mail: baltimo@ caltech.edu

${ }^{3}$ These authors contributed equally to this work.

Keywords: NF- $\kappa$ B; apoptosis; diabetes; T-cell development; autoimmunity

Abbreviations: NF- $\kappa \mathrm{B}$, nuclear factor $\kappa \mathrm{B}$; DN, double negative; DP, double positive; SP, single positive; TCR, T-cell receptor; MHC, major histocompatibility complex; $\mathrm{NADPH}$, reduced nicotinamide adenine dinucleotide phosphate; VDAC, voltage-dependent anion channel; FACS, fluorescent activated cell sorting; XIAP, X-linked inhibitor of apoptosis; TNFR1, tumor necrosis factor receptor 1; RAGE, receptor for advanced glycation end products; STZ, streptozotocin; NOD, non-obese diabetic; OVA, ovalbumin

Received 21.9.10; revised 08.11.10; accepted 10.11.10; Edited by M Piacentini; published online 17.12.10 
and in a processed form, p50), and NF- $\kappa \mathrm{B} 2$ (which occurs both as a precursor, p100, and as its processed product, p52). The c-REL, RelB and RelA proteins have a carboxy-terminal non-homologous transactivation domain, which strongly activates transcription from NF- $\kappa \mathrm{B}$-binding sites in target genes. In the resting state, $\mathrm{NF}-\kappa \mathrm{B}$ proteins exist as homo- or hetero-dimers in the cytoplasm bound to the inhibitory proteins of the $\mathrm{I} \kappa \mathrm{B}$ family. Activation usually occurs by signal-induced proteasomal degradation of the $\mathrm{I} \kappa \mathrm{B}$ proteins releasing the bound $\mathrm{NF}-\kappa \mathrm{B}$ proteins, which in turn translocates to the nucleus to initiate transcription of target genes including several anti-apoptotic genes. ${ }^{12}$ Developing thymocytes exhibits constitutive NF- $\kappa \mathrm{B}$ activity. Pre-TCR-induced $\mathrm{NF}-\kappa \mathrm{B}$ activation is important for delivering selective survival signals during thymocyte development. ${ }^{13}$ High glucose alone can activate $\mathrm{NF}-\kappa \mathrm{B}$ in several cell types including peripheral blood mononuclear cells, ${ }^{14}$ vascular smooth muscle cells, ${ }^{15}$ mesangial cells ${ }^{16}$ and hepatocytes. ${ }^{17}$ Although glucose is essential for thymocyte survival, ${ }^{2,18}$ evidence of glucosemediated NF- $\kappa \mathrm{B}$ activation in thymocytes during developmental selection is lacking.

In this study, we report the surprising finding that glucose is absolutely essential for the survival of the DP thymoma cell line, EL-4. We confirmed these findings in wild-type murine and human thymocytes. We discovered that glucose-mediated survival of DP thymocytes occurs through the upregulation of $\mathrm{NF}-\kappa \mathrm{B}$-dependent anti-apoptotic survival factors. Finally, we tested the physiologic relevance by evaluating the efficacy of central tolerance in the setting of hyperglycemia in vivo and found evidence of reduced apoptosis of potentially autoreactive T cells in diabetic mice. Taken together, we provide evidence for a central role for glucose in the effectiveness of thymic selection during T-cell maturation and the elimination of potentially autoreactive $T$ cells.

\section{Results}

EL-4 cell survival is dependent on glucose. In a screen of cell lines for glucose sensitivity, EL-4 cells demonstrated the unique property of acute dependence on the presence of glucose in the culture medium (Figure 1a). As early as $20 \mathrm{~h}$ after removing glucose, EL-4 cell numbers were nearly $90 \%$ diminished from starting amounts (Figure 1b). The addition of glucose to the culture media resulted in sustained dosedependent robust proliferation of EL-4 cells (Figure 1b). Inspection of the cellular morphology of EL-4 cells at various glucose concentrations showed that not only in the absence of glucose was there reduced cellularity (Figure 1c), but that morphologic features of apoptosis were evident as early as $12 \mathrm{~h}$ (Figure 1d). As a test for specificity of glucose effect and an osmotic control for glucose, we evaluated cultures in the absence of glucose supplemented with $30 \mathrm{mM}$ mannitol and found again reduced cellularity (Figure 1c). We confirmed the morphologic findings using Annexin $\mathrm{V}$ staining for a FACS evaluation of apoptosis. When we evaluated EL-4 cells, we found that glucose, even at a relatively low concentration $(10 \mathrm{mM})$, rescued EL-4 cells from apoptosis as evident by Annexin $V$ staining (Figure 1e). The 'zero' glucose condition we refer to is actually $0.5 \mathrm{mM}$ of glucose contributed by the $10 \%$ fetal bovine serum in culture medium.
DP thymocyte survival is dependent on glucose. EL-4 cells were derived from a mouse thymoma ${ }^{19}$ and express both $\mathrm{CD}^{+}{ }^{+}$and $\mathrm{CD}^{+}$on their surface (Supplementary Figure 1). We hypothesized that the observed glucose effect might be a unique feature of $\mathrm{CD} 4{ }^{+} \mathrm{CD}{ }^{+} \mathrm{DP}$ thymocytes. In our initial experiment, whole thymocytes taken from male C57BL/6 mice were prepared as a single-cell suspension and cultured for $24 \mathrm{~h}$ in vitro under several glucose concentrations. We confirmed that the majority of the thymocytes were indeed $\mathrm{CD}^{+}{ }^{+} \mathrm{CD} 8{ }^{+}$DP (Figure 2a). Thymocyte populations were then evaluated directly by FACS for evidence of apoptosis (7-AAD low/Annexin V high) (Figure 2b). Similarly to EL-4 cells, total thymocyte populations had a reduced numbers of cells undergoing apoptosis under hyperglycemic conditions (Figure 2c, top left). Interestingly, as we hypothesized, analysis of the sub-populations showed that the glucose-mediated partial rescue from apoptosis was limited to the $\mathrm{CD}^{+}{ }^{+} \mathrm{CD} 8{ }^{+} \mathrm{DP}$ cells (Figure $2 \mathrm{c}$, top right). The low level of apoptosis in the mature SP $\left(\mathrm{CD}^{+}{ }^{+}\right.$or $\left.\mathrm{CD}^{+}\right)$populations was unaffected by hyperglycemia (Figure 2c, bottom panels). Next, we evaluated a thymus from an aborted 16-week human fetus for the effect of glucose on apoptosis. We found that analogous to murine thymocytes, the human thymocytes also had reduced levels of apoptosis under hyperglycemic conditions (Figure 2d).

Hypoglycemia does not alter the pattern of proapoptotic factor activation. As apoptosis is a balance between the activation of death signals (pro-apoptotic) and the synthesis of survival (anti-apoptotic) factors, we probed both possibilities regarding the observed glucose effect. Given the convenience of the EL-4 cell line, we made observations in the cell line first, then confirmed them in the primary cells. Owing to the strict dependence of EL4 cells on glucose for survival, studies of glucose-induced transcriptional events in these cells at $6 \mathrm{~h}$ were performed under physiologic $(5 \mathrm{mM})$ and hyperglycemic $(30 \mathrm{mM})$ glucose concentrations. EL-4 cells grown in $0 \mathrm{mM}$ glucose concentration for $6 \mathrm{~h}$ yielded very low amounts of RNA, rendering gene expression studies difficult. As the induction of apoptosis was quick, occurring within hours after glucose withdrawal, we tested whether glucose-induced transcription and translation was involved in the survival of EL4 cells. Inhibition of new RNA or protein synthesis with Actinomycin-D or cycloheximide respectively, prevented $30 \mathrm{mM}$ glucose-mediated rescue of EL4 cells from apoptosis (Supplementary Figure 2).

In agreement with the FACS data showing apoptosis, we found that EL-4 cells under hypoglycemic conditions demonstrated caspase cascade activation as evident by the appearance of activated cleaved caspase 3 and PARP cleavage (Figure 3a, left). We confirmed the same effect in C57BL/6 thymocytes (Figure 3a, right). As thymocytes have a high level of apoptosis that is dependent on $\mathrm{Bim},{ }^{21}$ we tested for upregulation of Bim in the absence of glucose as a possible reason for the glucose-dependent phenotype. We found that low glucose conditions in both EL-4 cells (Figure 3b, left) and C57BL/6 thymocytes (Figure 3b, right) did not result in an augmented expression of Bim at the transcriptional level. Similarly, we did not observe an effect on the protein 
a

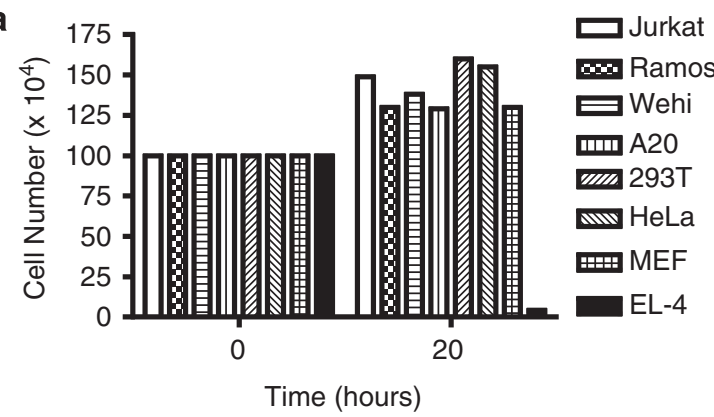

b

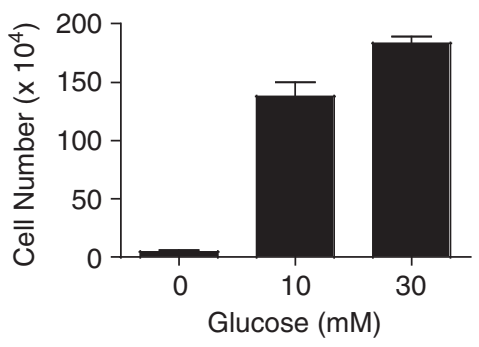

C

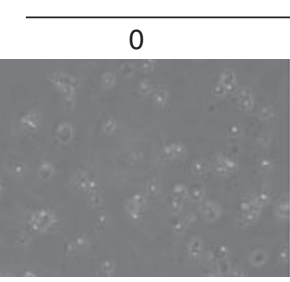

d

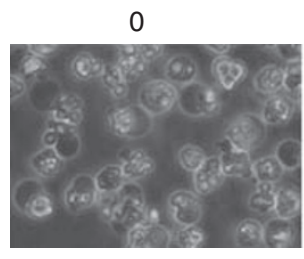

Glucose (mM)

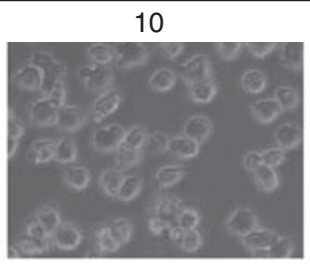

30

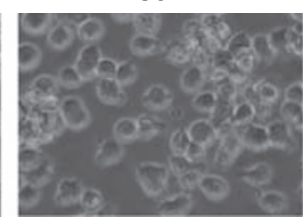

Mannitol (mM)

30
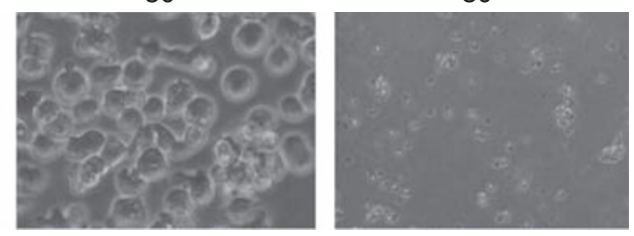

Glucose (mM)

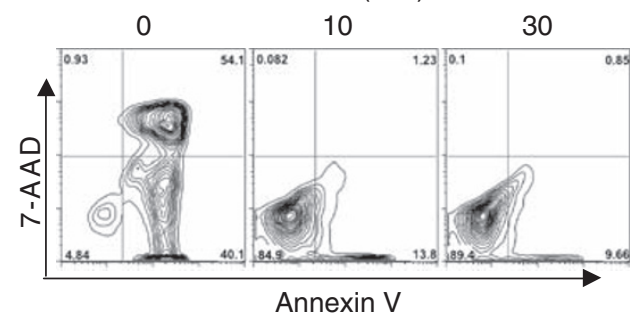

Figure 1 (a) EL-4 cells are unique in glucose dependence for survival. Equivalent numbers of starting cells $\left(1 \times 10^{6}\right.$ per well for the suspension cells in 12 -well plate and $1.5 \times 10^{5}$ per well in six-well plate for adherent cells) were seeded in media lacking glucose after having been growing in standard glucose containing media (RPMI $11.1 \mathrm{mM}$ glucose and DMEM $25 \mathrm{mM}$ glucose). After $24 \mathrm{~h}$, cell numbers were determined by Trypan blue exclusion. Data are representative of three independent experiments. (b-e) EL-4 cells are dependent on glucose for survival and proliferation. EL-4 cell cultures seeded with equivalent numbers of starting cells $\left(1 \times 10^{6}\right)$ in 12 -well plate in RPMI with indicated final concentrations of glucose or mannitol. After $20 \mathrm{~h}$, cell number determined by Trypan blue exclusion (b) and $24 \mathrm{~h}$ later by visual inspection on a Nikon Diaphot 300 phase contrast microscope (NIKON, Melville, NY, USA) at $\times 400$ final magnification and photographed using a SPOT digital camera and software (Sterling Heights, MI USA). (d) Evaluation of EL-4 cells as described above at $12 \mathrm{~h}$ showed apoptotic morphology. (e) EL-4 cells were cultured at the indicated glucose concentrations for $12 \mathrm{~h}$ and then evaluated by FACS for evidence of early apoptosis (7-AAD negative/Annexin V positive). Data are representative of four independent experiments

expression of Bim as a function of glucose concentration in either EL-4 (Figure 3c, left) or C57BL/6 thymocytes (Figure 3c, right). We also checked another proapoptotic molecule $\mathrm{Bax}^{22}$ in thymocytes and found no significant effect of glucose concentration in its expression level (Figure $3 c$, right).

\section{Hyperglycemia results in expression of anti-apoptotic} factors. As we did not observe a hypoglycemic augmentation of pro-apoptotic factors, we tested the hypothesis that the observed amelioration from apoptosis in the setting of hyperglycemia was due to an increased production of survival factors. Again, using the convenience of EL-4 cells as our guide, we found an acute upregulation of several wellcharacterized anti-apoptotic gene transcripts in the setting of hyperglycemia (30 mM glucose) (Figure 4a). As shown in Figure $4 b$, wild-type thymocytes responded to hyperglycemia in an analogous fashion with an acute upregulation of survival factors (Figure $4 \mathrm{~b}$ ). We were able to confirm at the protein level that under hyperglycemic conditions, Bcl-2 and XIAP are upregulated in both EL-4 cells (Figure 4c) and C57BL/6 thymocytes (Figure $4 d$ ). To exclude the possibility that the reduced protein levels of survival factors under hypoglycemic conditions were due to degradative effects of caspases, we performed tests in presence of caspase inhibitors. We found in EL-4 cells that caspase blockade (with z-VAD-fmk) in low glucose conditions did not result an augmented detection of XIAP, which is a known caspase 3 substrate ${ }^{23}$ (Figure 4e).

Hyperglycemia-induced rescue from apoptosis is mediated by NF- $\kappa$ B p65. The anti-apoptotic genes that we found to be upregulated under hyperglycemic conditions (Figure 4) are known to be activated by NF- $\kappa B^{24}$ In particular, expression of several survival factors is driven by p65 or c-Rel. ${ }^{25,26}$ Furthermore, glycosylation of p65 under hyperglycemic condition has been shown to sustain its activity. ${ }^{16}$ To determine the role of each of these NF- $\kappa \mathrm{B}$ sub-units in glucose-mediated thymocyte survival, we performed experiments using thymocytes from c-Rel knockout and $\mathrm{p} 65^{-1-} \mathrm{TNFR}^{-1-}$ double knockout animals. Mice lacking only p65 are embryonic lethal but they survive on a TNFR $1^{-1-}$ background. $^{27}$ Therefore, as a control, 
a

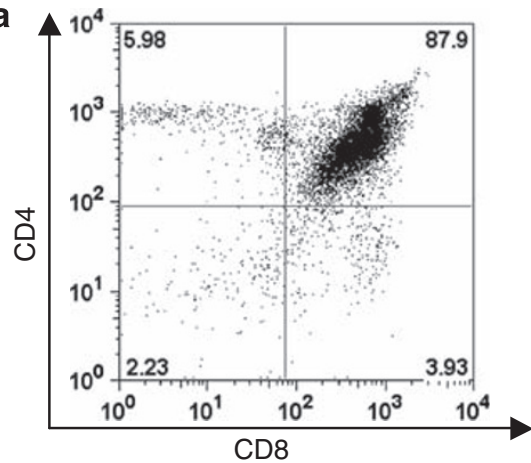

C Total Thymocytes

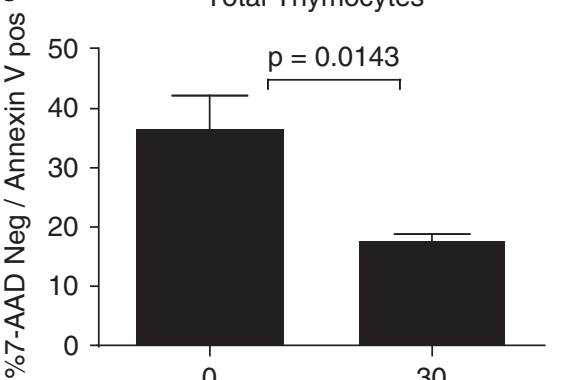

Glucose (mM)

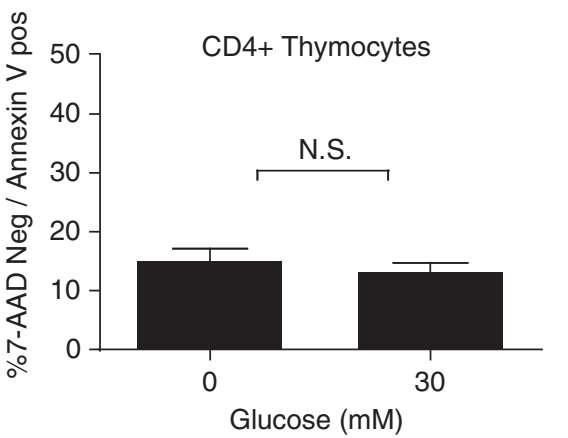

b $\quad \mathrm{CD} 4+\mathrm{CD} 8+$

CD4+

CD8+
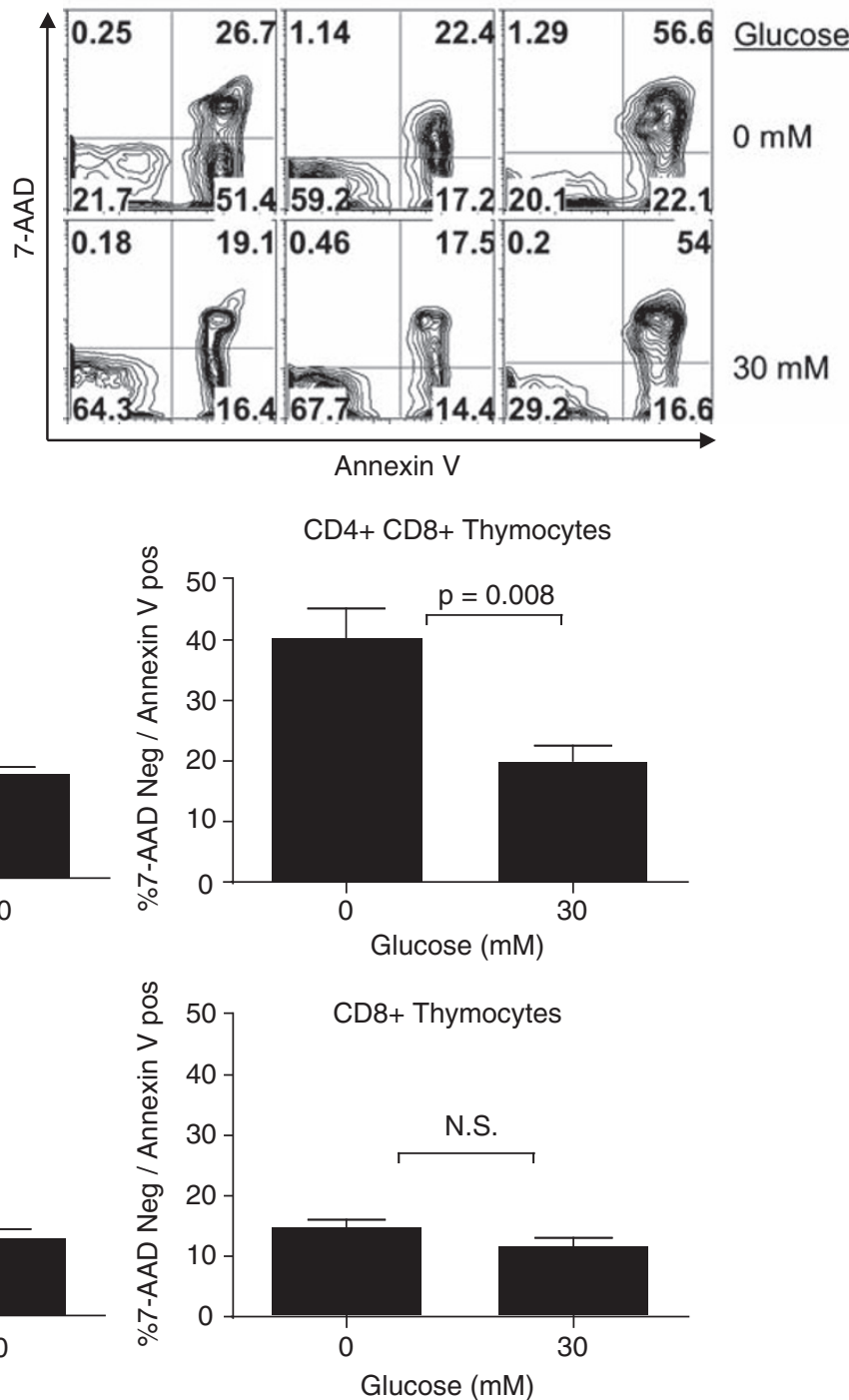

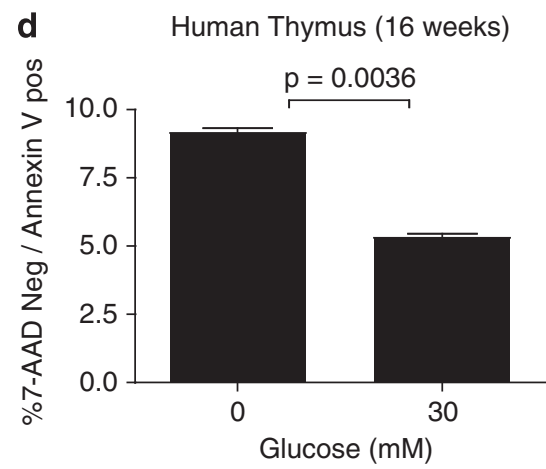

Figure 2 Enhanced thymocyte glucose-mediated survival is restricted to the CD4 + CD8 + population. (a and $\mathbf{b})$ Single-cell suspensions were prepared from male $\mathrm{C} 57 \mathrm{BL} / 6$ thymuses and cultured for $24 \mathrm{~h}$ in vitro with the indicated concentration of glucose. The cell suspensions were collected and stained for CD4, CD8, 7-AAD and Annexin V for FACS evaluation. Indicated sub-populations were evaluated for cells in the early stages of apoptosis (7-AAD negative/Annexin V positive). (c) Composite thymic subpopulation evaluations from male C57BL/6 $(n=4)$. (d) Single-cell suspension was prepared from a human fetal thymus (gestational age $=16$ weeks) isolated immediately following the surgical termination. The cell suspension was cultured for $24 \mathrm{~h}$ in vitro with the indicated concentration of glucose, subsequently stained for CD4, CD8, 7-AAD and Annexin V for FACS evaluation for early stage apoptosis (7-AAD negative/Annexin V positive). Statistical difference determined by two-tailed unpaired Student's $t$-test (N.S., nonsignificant) 


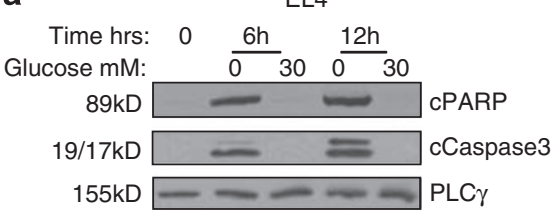

b

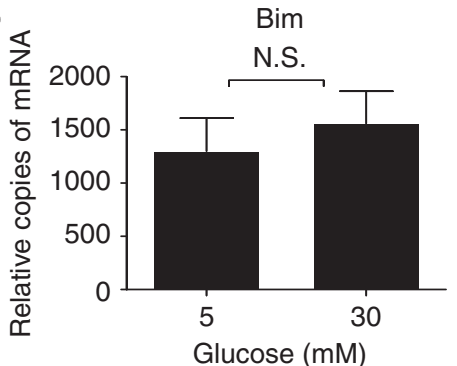

C

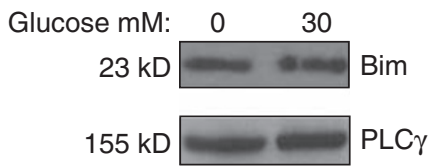

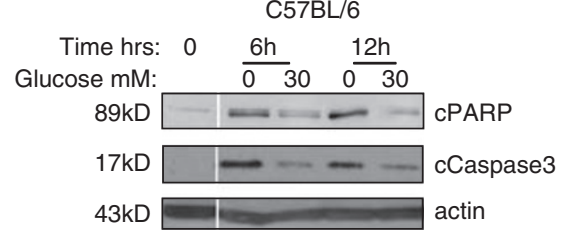
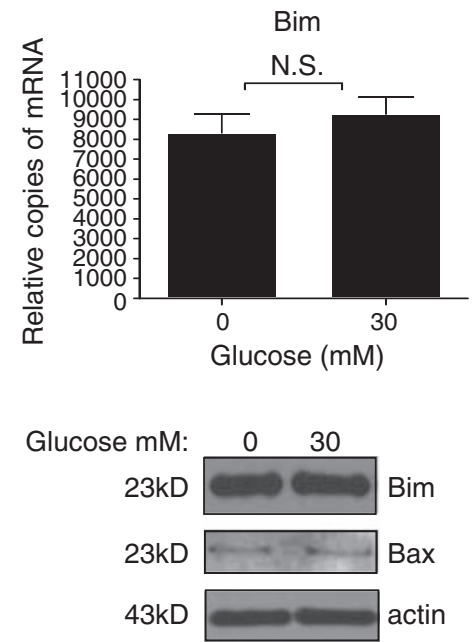

Figure 3 Glucose inhibits thymocyte caspase activation, but not through altered tonic Bim levels. (a) EL-4 cells (left) or single-cell suspension from male C57BL/6 thymocytes ${ }^{20}$ were cultured for the indicated times and glucose concentrations before total cellular protein isolation. Western blot membranes were probed for cleaved PARP (cPARP), cleaved caspase 3 (cCaspase3). Phospholipase $\mathrm{C} \gamma$ (PLC $\gamma$ ) and actin were used as the loading controls. (b) Total RNA was isolated from EL-4 cells (left) or single-cell suspension from male C57BL/ 6 thymocytes ${ }^{20}$ that had been cultured for the 6 h at indicated glucose concentrations. As the EL 4 cells were unable to survive at 0 mM glucose, the cells were cultured overnight at $5 \mathrm{mM}$ glucose and then treated with $30 \mathrm{mM}$ glucose. Quantitative RT-PCR was performed in triplicate and relative abundance of Bim transcripts calculated. Statistical difference determined by two-tailed unpaired Student's t-test (N.S., nonsignificant). (c) Total cellular proteins were isolated as above. Western blot membranes from EL-4 cells were probed for Bim and PLC $\gamma$ (left). Western blot membranes from C57BL/6 thymocytes were probed from Bim and Bax ${ }^{20}$

we evaluated thymocytes from TNFR1 ${ }^{-1-}$ animals. Thymo-

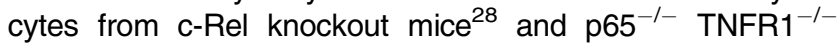
double knockout mice were evaluated in vitro for glucosemediated survival. We found that under hyperglycemic conditions, thymocytes lacking c-Rel demonstrated the previously documented reduced level of apoptosis (Figure 5a). In contrast, $\mathrm{p} 65^{-1-} \mathrm{TNFR}^{-1-}$-deficient thymocytes did not show enhanced survival with high glucose (Figure $5 \mathrm{~b}$ ). The control TNFR1 ${ }^{-l-}$ thymocytes behaved just like the wild-type mice showing that the observed glucose-mediated survival is p65 dependent (Supplementary Figure 3).

We examined the expression level of survival factors in p65 ${ }^{-1-}$ TNFR1 $1^{-1-}$ thymocytes. In line with the lack of survival as observed by FACS analysis, glucose-induced upregulation of anti-apoptotic genes was dramatically reduced in these cells. A modest and not significant increase in the expression of some survival factors in $\mathrm{p} 65^{-/-}$TNFR $1^{-/-}$thymocytes was evident, perhaps through a compensatory pathway (Figure 5c). To examine if the lack of induction of antiapoptotic genes in NF- $\kappa \mathrm{B}$ p65 knockout mice was due to a defect in glucose responsiveness of these cells, we examined the expression of glucose transporters in these cells. Glucose is known to enhance the expression of glucose transporters in thymocytes. ${ }^{18}$ We observed a similar induction of Glut1-Glut4 in control and NF- $\kappa$ B p 65 knockout thymocytes (Supplementary Figure 4). Glucose also upregulates the expression of the receptor for advanced glycation end products (RAGE) and the RAGE pathway is known to promote apoptosis. ${ }^{29,30}$ Like glucose transporters, RAGE expression was comparable in control and NF- $\kappa \mathrm{B}$ p65 knockout thymocytes (Supplementary Figure 4). Thus, it appears that it is a lack of survival factors in the knockout cells that lead to their apoptosis and not a lack of glucose transporters or RAGE.

Hyperglycemia is associated with general NF- $\kappa$ B activation and upregulation of markers of inflammation. ${ }^{31}$ Consistently, as shown in Figure $5 d$, we found that under hyperglycemic conditions $\mathrm{I} \kappa \mathrm{B} \alpha$ and IP-10 transcripts are increased in the wild-type thymocytes. Furthermore, we found that phosphorylated AKT was increased with high glucose (Figure 5e, top panel) perhaps providing the link between hyperglycemia, $\mathrm{NF}-\kappa \mathrm{B}$ and the expression of survival factors. Total AKT protein level was comparable in the low and high glucosetreated cells (Figure 5e, middle panel) while PARP was found cleaved in the low glucose condition (Figure5e, bottom panel).

Hyperglycemia results in reduced effectiveness of central tolerance. Our observations to this point have been in vitro. To test for physiologic relevance, we sought to evaluate the in vivo survival of thymocytes in the setting of hyperglycemia. DP thymocytes mature to SP $\left(\mathrm{CD}^{+}{ }^{+}\right.$or $\mathrm{CD}^{+}$) cells. During this transition, if thymocytes receive a strong signal through their TCR, they will undergo apoptosis thus eliminating thymocytes ( $T$ cells) that are potentially autoreactive. $^{32}$ In mice with CD4 ${ }^{+} \mathrm{T}$ cells transgenic for an 
a
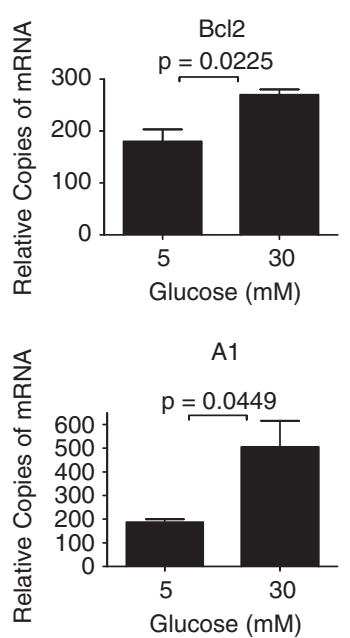

EL4

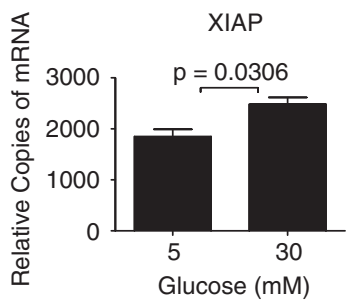

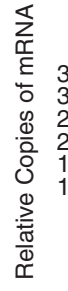

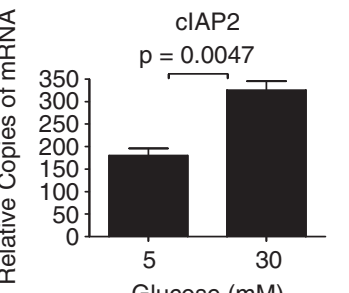

b
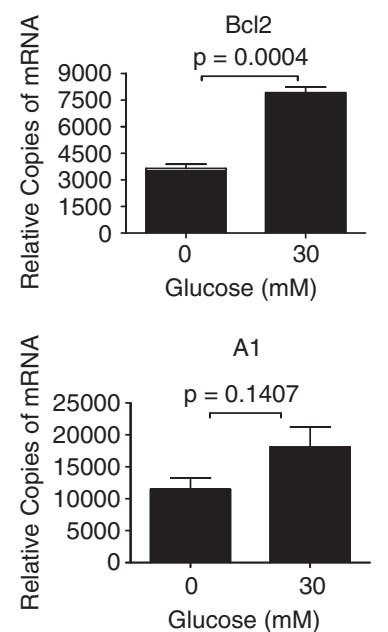

C57BL/6
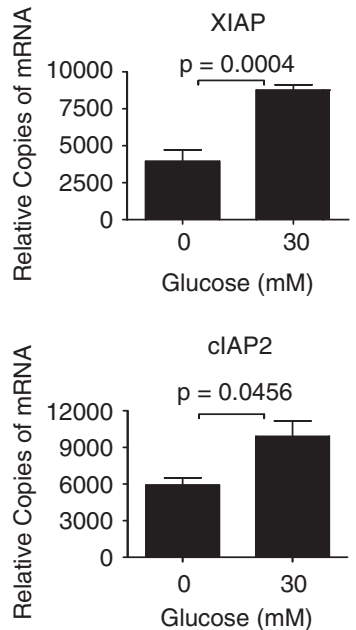

c

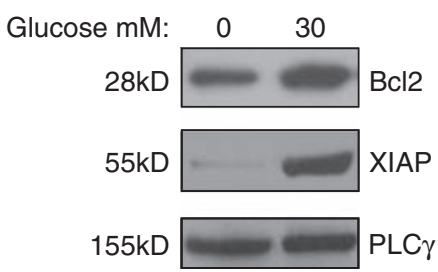

d Glucose mM:

$28 \mathrm{kD}$

$55 \mathrm{kD}$

$43 \mathrm{kD}$

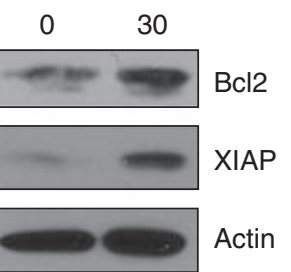

e

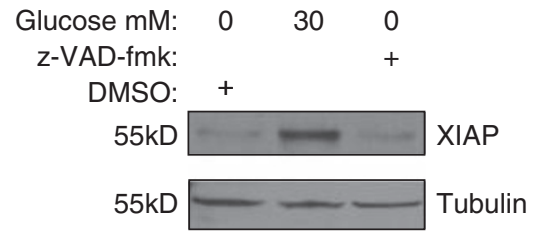

Figure 4 High glucose causes upregulation of anti-apoptotic genes. Total RNA was isolated from EL-4 cells (a) or single-cell suspension from male C57BL/6 thymus (b) grown and treated as in Figure 3b. Quantitative RT-PCR was performed in triplicate and relative abundance of Bcl2, XIAP, A1 and clAP transcripts calculated. Statistical difference determined by two-tailed unpaired Student's t-test (NS, nonsignificant). EL-4 cells (c) or single-cell suspension from male C57BL/6 (d) were cultured for 10 and $24 \mathrm{~h}$, respectively at the indicated glucose concentrations before total cellular protein isolation. Western blot membranes were probed for Bcl2 and XIAP. Actin and Phospholipase $\mathrm{C} \gamma(\mathrm{PLC} \gamma)$ were used as loading controls. (e) EL-4 cells were cultured for $10 \mathrm{~h}$ under the indicated conditions ( $\mathrm{z}$-VAD-fmk at a final concentration of $50 \mu \mathrm{M})$ before total cellular protein isolation. Western blot membranes were probed for XIAP and tubulin was used as a loading control

ovalbumin-specific TCR (OT2), exposure to OVA in vivo causes deletion of the transgenic TCR-bearing $T$ cells in the thymus. ${ }^{33}$ In this context, OVA is acting as a surrogate selfantigen. To test the effect of hyperglycemia on thymocyte survival, we used streptozotocin (STZ) to induce diabetes and therefore hyperglycemia in OT2 and wild-type C57BL/6 control mice. All the STZ-treated mice in the control (Figure 6a, left) and OT2 (Figure 6a, right) groups developed significant hyperglycemia. Once the mice were hyperglycemic, all mice were given OVA i.p. and euthanized $48 \mathrm{~h}$ later. Evaluation of the thymocytes in OT2 mice showed an increased survival of the DP (Figure 6b, right) and CD4 ${ }^{+}$ SP (Figure 6c, right) populations in the hyperglycemic mice. We did not observe increased survival in the $\mathrm{CD}^{+} \mathrm{SP}$ population in OT2 mice (Figure 6d, right). Not surprisingly, the effect of OVA exposure or hyperglycemia on the survival of any thymocyte population in wild-type controls was not observed because of the lack of specific antigenic selection pressure (Figures 6b-d, left).

\section{Discussion}

Our results are part of a growing body of evidence showing that glucose has an important role in cellular fate beyond that as a bioenergetic substrate. We made the serendipitous observation that EL-4 thymoma cells have a unique reliance on glucose for survival. We confirmed in primary murine thymocytes that the dependence on glucose for survival is a feature of $\mathrm{CD} 4{ }^{+} \mathrm{CD} 8{ }^{+}$DP thymocytes, but not of DN or SP thymic sub types. Furthermore, we found a similar reliance of DP human thymocytes on glucose for survival. We attribute the molecular mechanism to a glucose-induced expression of anti-apoptotic factors that is dependent on NF- $\kappa \mathrm{B}$ p65. Finally, we tested our in vitro observations for in vivo relevance and found that induced diabetic mice have significantly inhibited effective central tolerance.

Apoptosis has a central role in shaping a T-cell repertoire that is both functional and non-self reactive. As a consequence, most thymocytes are destined to die. It has previously been appreciated in chicken embryonic thymic organ culture that hyperglycemia activates thymocyte metabolism and decreases thymocyte apoptosis, ${ }^{18}$ but similar evidence in a mammalian system is lacking. Glucose enhances cellular metabolism and the production of metabolites like NADPH that can inhibit caspases. ${ }^{10}$ Further, hexokinase itself or metabolites that are produced by hexokinase activation bind to VDAC in mitochondria thus blocking cell death. ${ }^{6}$ Our findings suggest an additional mode of glucose-mediated protection of thymocytes from apoptosis through activation of the NF- $\kappa \mathrm{B}$ pathway.

Changes in cellular apoptotic fate can be influenced by either an increase in death signaling (pro-apoptosis) or by the action of survival (anti-apoptotic) factors. The role that glucose may be having on this balance in thymocytes 

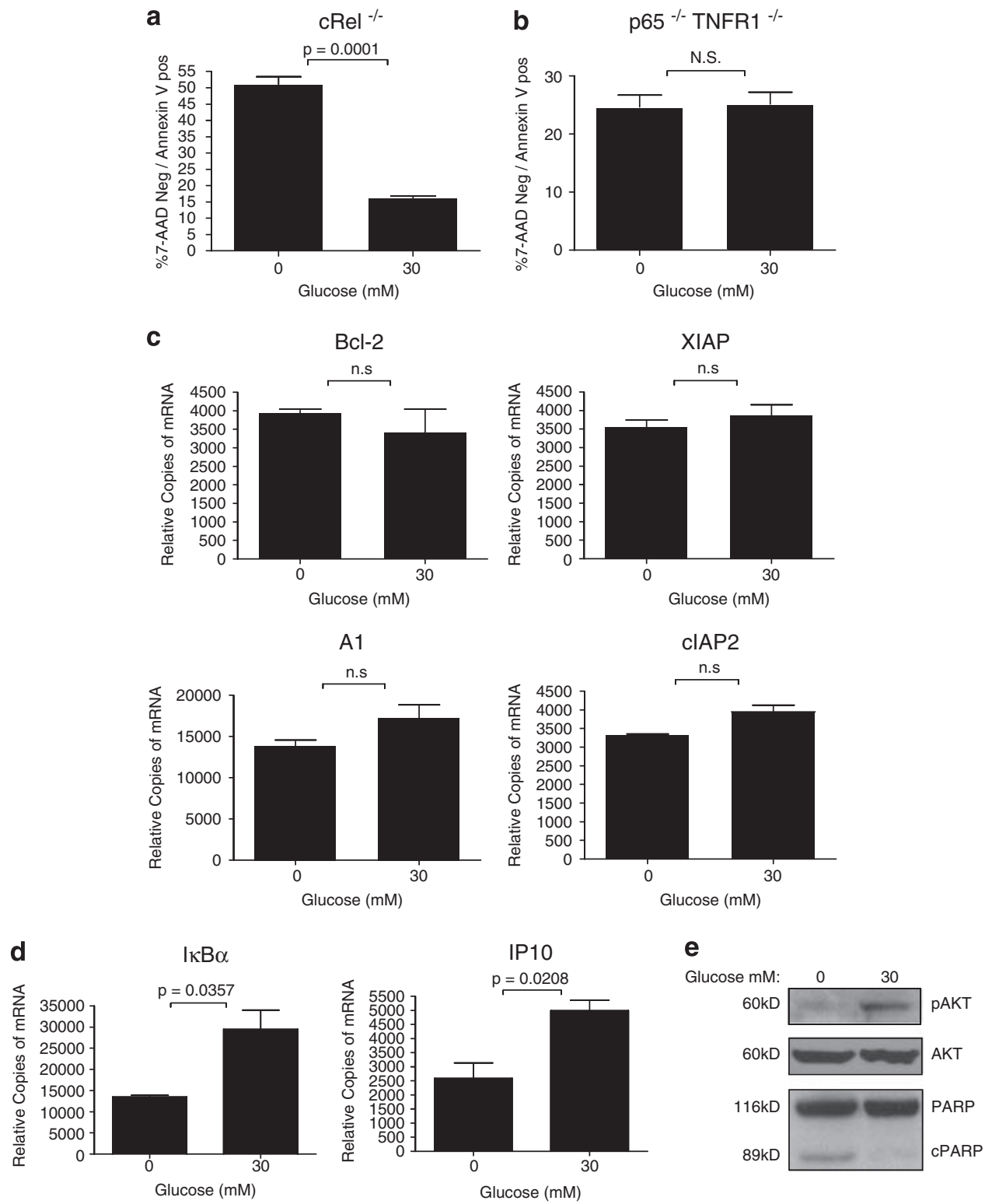

Figure 5 Anti-apoptotic glucose effect on thymocytes is dependent on p65 NF- $\kappa \mathrm{B}$. Single-cell suspensions were prepared from (a) male c-Rel ${ }^{-/-} \mathrm{C} 57 \mathrm{BL} / 6(n=4)$ or (b) male p65 $5^{-1-}$ TNFR1 ${ }^{-1-}(n=2)$ thymuses and cultured for $24 \mathrm{~h}$ in vitro with the indicated concentration of glucose. The cell suspensions were collected and stained for $\mathrm{CD} 4, \mathrm{CD} 8,7-\mathrm{AAD}$ and Annexin V for FACS evaluation. CD4 + CD8 + population was evaluated for early stages of apoptosis (7-AAD negative/Annexin V positive). Statistical difference determined by two-tailed unpaired Student's $t$-test (N.S., nonsignificant). (c) Total RNA was isolated from male p65 ${ }^{-1-}$ TNFR $^{-1-}$ thymic single-cell suspension cultured for $6 \mathrm{~h}$ at the indicated glucose concentrations. Quantitative RT-PCR was performed in triplicate and relative abundance of Bcl2, XIAP, A1 and cIAP transcripts calculated. Statistical difference determined by two-tailed unpaired Student's t-test (N.S., nonsignificant). (d and $\mathbf{e})$ High glucose induces general activation of the NF- $\kappa$ B pathway and phosphorylation of AKT (pAKT). (d) Total RNA was isolated from single-cell suspension cultured in vitro at the indicated glucose concentrations from male $\mathrm{C} 57 \mathrm{BL} / 6$ thymus and the relative abundance of $\mathrm{I}_{\kappa \mathrm{B}} \alpha$ and IP10 transcripts determined by $\mathrm{qPCR}$. (e) Total protein was isolated from the thymocyte cultures above and western blot was probed for PAKT, AKT and PARP

could be either through hypoglycemia-induced pro-apoptosis factors, or through hyperglycemic-induced survival factors. Glucose-mediated upregulation of anti-apoptotic genes has been reported previously in vascular smooth muscle cells ${ }^{15}$ and $\mathrm{Bcl}-2$ (a NF- $\kappa \mathrm{B}$-dependent gene) has also been shown to be upregulated in NOD thymocytes. ${ }^{34}$ In line with these previous observations, we also found a glucose-dependent increase in Bcl-2 and the IAP family of anti-apoptotic molecules under hyperglycemic conditions.
Interestingly, transgenic mice over expressing XIAP or Bcl2 have decreased thymocyte apoptosis. ${ }^{20,35}$ Conversely, knockouts of the pro-apoptotic protein Bim (suggested to be crucial for thymocyte apoptosis) also have decreased apoptosis of thymocytes. ${ }^{21}$ However, we did not find an increase in Bim mRNA or protein under hypoglycemic conditions. Similarly, we did not observe an increase in Bax protein under hypoglycemic conditions. Our results suggest that apoptosis of thymocytes under hypoglycemia occurs by 
a

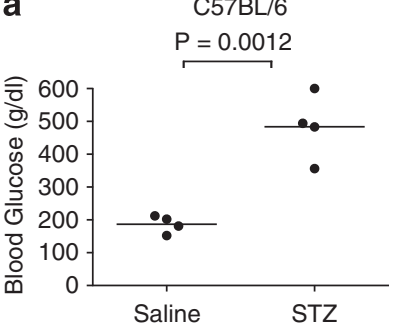

C
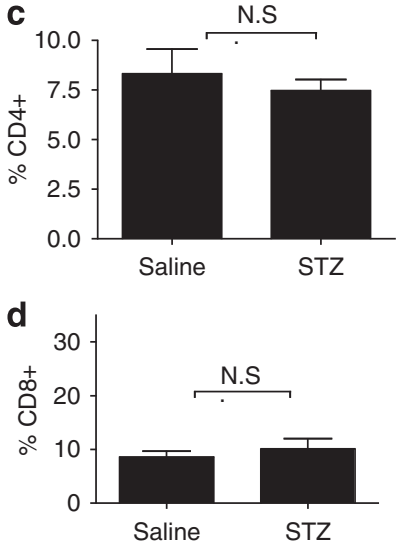

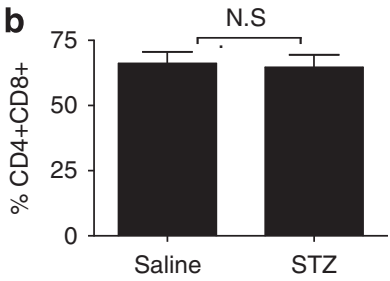

OT2
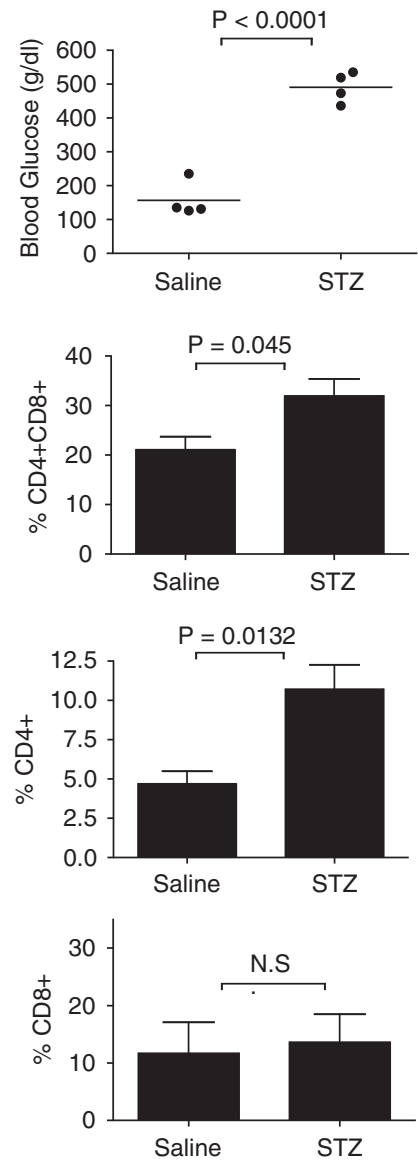

Figure 6 Hyperglycemia results in reduced deletion of antigen-specific transgenic TCR thymocytes when challenged with antigen. C57BL/6 and C57BL/6 - OT2 were treated with STZ $(n=4)$ or saline $(n=4)$. After 3 weeks, the mice were given $10 \mathrm{mg} / \mathrm{kg}$ ovalbumin i.p. The mice were euthanized $24 \mathrm{~h}$ later. Blood from cardiac puncture was evaluated for glucose concentration (a). Thymic single-cell suspensions were created from the above mice, surface stained for CD4 and CD8, and sub-populations (b-d) evaluated by FACS. Statistical difference determined by two-tailed unpaired Student's $t$-test (N.S., nonsignificant)

lack of induction of anti-apoptotic genes rather than by upregulation of pro-apoptotic molecules.

Details of the molecular impact of hyperglycemia are emerging. Among the most intriguing are the potential molecular influence of glycosylation on transcription factors, including NF- $\kappa \mathrm{B}$ family members. The O-GlcNAcylation of p65 decreases the affinity of the interaction with $I_{\kappa} \mathrm{B}$ and consequently increases the activity of p65 under hyperglycemic conditions. ${ }^{16,36}$ Downstream consequences of chronic p65 activation include the upregulation of several pro-inflammatory, NF- $\kappa \mathrm{B}$-dependent genes that can modulate apoptosis. In addition to activation of NF- $\kappa \mathrm{B}$-dependent transcription, high glucose might also induce direct posttranslational modification of some anti-apoptotic proteins and stabilize their expression level.

$\mathrm{NF}-\kappa \mathrm{B}$ is also activated by the AKT pathway, ${ }^{37}$ which is activated under hyperglycemic conditions and is known to couple glucose metabolism with cell survival. ${ }^{38} \mathrm{We}$ also observed AKT phosphorylation under hyperglycemic conditions (Figure 5e) and conversely, phosphorylated AKT was almost undetectable when glucose was withdrawn from the cell culture. Collectively, our data suggest the possibility of glucose-induced survival signaling in thymocytes through the AKT pathway of NF- $\kappa$ B activation.

We asked if our in vitro observations had a physiologic relevance in vivo to developing thymocytes. We induced hyperglycemic conditions in vivo through STZ-mediated diabetes. OT2 mice are transgenic for CD4 $+\mathrm{T}$ cells that bear a TCR specific for OVA. When these mice are exposed to OVA in vivo, thymocytes with TCRs specific for OVA are deleted. In this setting, OVA is acting as a self-antigen and OT2 T cells are potentially auto-reactive. Amazingly, we found that under diabetic conditions, the ability of OT2 mice to clear these potentially auto-reactive thymocytes was significantly inhibited compared with the non-diabetic controls. Further, this effect seems to be antigen specific because in the wild-type mice, induction of diabetes did not significantly alter the thymocyte populations compared with non-diabetic wild-type controls. It should be noted that while the glucosemediated effect is observable in vitro, the survival advantage is subtle and is only observable in vivo under the extremely biased situation of a TCR transgenic mouse under antigenic selection.

Our findings pose an interesting conundrum regarding autoimmune diabetes, type I or latent autoimmune diabetes of the adult. Is it the hyperglycemia that develops as a consequence of destruction of pancreatic beta cells ${ }^{39}$ that exacerbates the onset of the disease or does the resulting hyperglycemia predispose to other autoimmune conditions? This study does not attempt to dissect this complex interplay, but our data do indicate that the presence of excessive glucose from any source can have a detrimental impact on the ability of developing thymocytes to be appropriately selected. Although the composition of the T-cell repertoire evolves throughout life, a peak period of susceptibility to hyperglycemiamediated interference would be during maximal thymic selection that occurs during fetal life through early childhood. Thus, gestational diabetes as well as early onset hyperglycemia from any cause could potentially predispose these individuals to autoimmunity.

In light of the fact that diabetes has reached epidemic proportions and that the developmental effects of diabetes are relatively unexplored, our results point to a crucial intersection. Specifically, hyperglycemic conditions result in a skewing of T-cell development in thymus. NF- $\kappa \mathrm{B}$ is considered as a primary target for treating inflammation and autoimmunity. We show here that NF- $\kappa$ B has an additional role in the preceding step of autoimmune conditions by enhancing the survival of thymocytes that are destined to die. Our findings also open the possibility of targeting thymus NF- $\kappa \mathrm{B}$ as a means of therapy early in life to ameliorate the immune and inflammatory complications of hyperglycemia.

\section{Materials and Methods}

Materials. Glucose and STZ were purchased from Sigma (St Louis, MO, USA). The antibodies for PARP, phospholipase-C $\gamma$, actin, c-Rel, p65, Bax, XIAP, and tubulin were from Santa Cruz (Santa Cruz, CA, USA). Antibodies against cleaved caspase3, phospho AKT and Bcl2 were from Cell Signaling Technology (Danvers, MA, USA). Anti-Bim antibody was from Enzo (Plymouth Meeting, PA, USA). 
Fluorescent antibodies used for FACS (CD4, CD8), FITC conjugated Annexin V and 7-AAD cell viability staining solution were purchased from Biolegend (San Diego, CA, USA). Ficoll-paque PLUS was from GE Healthcare (Piscataway, NJ, USA).

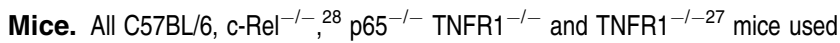
were 5-9 week old males. Five-week-old male C57BL/6 OT2 mice were purchased from The Jackson Laboratory (Bar Harbor, ME, USA). Mice were housed and handled in accordance with National Institutes of Health Guidelines under Institutional Animal Care and used committee-approved protocols.

Cells. Mouse thymoma EL-4 cell line was generously provided by Dr. Ellen Rothenberg. EL-4 and the B-lymphoblastoid cell lines WEHI and A20 were maintained in C/10 RPMI media (RPMI 1640 supplemented with 1\% nonessential amino acids, $4 \mathrm{mM}$ L-glutamine, $10 \mathrm{mM}$ HEPES buffer, $1 \mathrm{mM}$ sodium pyruvate, $100 \mathrm{U} / \mathrm{ml}$ penicillin/streptomycin, $0.05 \mathrm{mM}$ 2-mercaptoethanol, and $10 \%$ heatinactivated fetal bovine serum). Jurkat and Ramos cells were maintained in complete RPMI media (1\% penicillin/streptomycin, $1 \%$ L-glutamine and $10 \%$ fetal bovine serum). In all, 293T, HeLa and mouse embryonic fibroblast cells were grown in DMEM complete media (100 U/ml penicillin/streptomycin, $4 \mathrm{mM} \mathrm{L-glutamine} \mathrm{and}$ $10 \%$ fetal bovine serum). Thymocytes were prepared by passing the isolated thymus through $70 \mu \mathrm{m}$ cell strainer to obtain single-cell suspension.

Human tissue. Human thymic tissue was obtained for evaluation under a UCLA Office of the Human Research Protection Program and California Institute of Technology Institutional Review Board-approved protocol. The human thymic tissue was obtained from a 16-week fetus undergoing elective pregnancy termination at Ronald Regan UCLA Medical Center. The tissue was washed with PBS and then prepared into a single-cell suspension by passing through a $70 \mu \mathrm{m}$ cell strainer (BD Biosciences, San Diego, CA, USA). The thymocytes were cultured under the same conditions as murine primary thymocytes above.

STZ-induced diabetic mouse model. STZ treatment was performed as described. ${ }^{40}$ Briefly, mice were deprived of food for $4 \mathrm{~h}$ and then injected with $40 \mathrm{mg} / \mathrm{kg}$ of STZ in citrate buffer per day for 5 consecutive days at $24-h$ interval. During the treatment, mice were provided ad lib with $10 \%$ sucrose in drinking water. Blood glucose level of the citrate buffer injected control and STZ-treated mice were monitored using Accu-chek advantage glucose meter (Roche Diagnostics, Indianapolis, IN, USA) after euthanasia.

Flow cytometry. Cells were surface labeled in PBS with $1 \%$ BSA for 15 min at $4{ }^{\circ} \mathrm{C}$ followed by a wash. For apoptosis evaluations the cells were resuspended in Annexin V-binding buffer (Biolegend) according the manufacturer's instructions and stained from Annexin V and 7-AAD. Analysis was performed on a BD FACS Calibur with post-acquisition analysis performed with Flowjo (Treestar, Ashland, OR, USA).

Immunoblotting. For checking the anti-apoptotic protein levels at the time of FACS analyses, thymocytes were cultured in 0 and $30 \mathrm{mM}$ glucose containing media for $24 \mathrm{~h}$. Live cells were isolated by ficoll gradient centrifugation at $450 \mathrm{~g}$ for $30 \mathrm{~min}$ at $25^{\circ} \mathrm{C}$. Interphase cells were collected washed with PBS. Cells were lysed on ice for $30 \mathrm{~min}$ in the lysis buffer (11 mM Sodium phosphate $\mathrm{pH} 7.4,150 \mathrm{mM} \mathrm{NaCl}$, $0.1 \%$ SDS, $0.5 \%$ sodium deoxycholate, $1 \%$ Triton $\mathrm{X}-100$ and $1 \mathrm{X}$ complete protease inhibitor cocktail). The lysate was boiled with LDS sample buffer (Invitrogen, Carlsbad, CA, USA) and resolved in Novex 4-12\% Bis-Tris gels (Invitrogen), transferred to nitrocellulose membrane, probed using relevant antibodies and detected using the Supersignal West Pico Chemiluminescent Substrate System (Pierce, Rockford, IL, USA).

RNA preparation and quantitative real-time PCR. DNA-free RNA was prepared using the RNeasy Mini Kit according to the manufacturer's instructions (Qiagen, Valencia, CA, USA). cDNA was synthesized from 0.5 to $1.0 \mu \mathrm{g}$ total RNA using random hexamers (Promega, Madison, WI, USA) and Superscript III reverse transcriptase first-strand synthesis kit (Invitrogen). Quantitative real-time PCR using cDNA corresponding to 10-20 ng of total RNA was performed using SYBR GREEN PCR Master Mix (Kapa Biosystems, Woburn, MA, USA) in a real-time PCR machine (Realplex, Eppendorf, Hamburg, Germany). The results obtained for individual genes were normalized to the ribosomal protein Rpl32. The primers used for PCR were designed from Primer Bank (http://pga.mgh.harvard.edu/primerbank/index.html) or Oligoperfect (http://tools.invitrogen.com/content.cfm?pageid = 9716) and their sequences are given in Supplementary Table 1.
Statistical analysis. Differences were statistically analyzed using unpaired Student's $t$-test or analysis of variance with Bonferroni/Dunn post test. Analysis was accomplished with PRISM (Graphpad, La Jolla, CA, USA).

\section{Conflict of interest}

The authors declare no conflict of interest.

Acknowledgements. We thank the family planning division of the UCLA Department of Obstetrics and Gynecology as well as Dr. Sarah Dry of the UCLA Department of Pathology and Laboratory Medicine for assistance in isolating the human thymic tissue. c-Rel knockout animals were kindly provided by Dr. Hsiou-Chi Liou, Weill Medical College of Cornell University, New York, USA. We acknowledge the assistance Jorge Mata and Scott Washburn of the Caltech Office of Laboratory Resources. PR is supported by Supported by the National Institutes of Health 2R01GM039458 to DB. DA Kahn is supported by the National Institutes of Health Building Interdisciplinary Research Careers in Women's Health (BIRCWH) center at UCLA (K12 HD001400).

1. Patterson CC, Dahlquist GG, Gyurus E, Green A, Soltesz G. Incidence trends for childhood type 1 diabetes in Europe during 1989-2003 and predicted new cases 2005-20: a multicentre prospective registration study. Lancet 2009; 373: 2027-2033.

2. Ciofani M, Zuniga-Pflucker JC. Notch promotes survival of pre-T cells at the beta-selection checkpoint by regulating cellular metabolism. Nat Immunol 2005; 6: 881-888.

3. Hayday AC, Pennington DJ. Key factors in the organized chaos of early $T$ cell development. Nat Immunol 2007; 8: 137-144.

4. Zhang N, Hartig H, Dzhagalov I, Draper D, He YW. The role of apoptosis in the development and function of T lymphocytes. Cell Res 2005; 15: 749-769.

5. Greiner EF, Guppy M, Brand K. Glucose is essential for proliferation and the glycolytic enzyme induction that provokes a transition to glycolytic energy production. J Biol Chem 1994; 269: 31484-31490.

6. Jacobs SR, Rathmell JC. Lymphocyte selection by starvation: glucose metabolism and cell death. Trends Immunol 2006; 27: 4-7.

7. Yu Q, Erman B, Bhandoola A, Sharrow SO, Singer A. In vitro evidence that cytokine receptor signals are required for differentiation of double positive thymocytes into functionally mature CD8+ T cells. J Exp Med 2003; 197: 475-487.

8. Bolanos JP, Almeida A, Moncada S. Glycolysis: a bioenergetic or a survival pathway? Trends Biochem Sci 2010; 35: 145-149.

9. Rathmell JC, Fox CJ, Plas DR, Hammerman PS, Cinalli RM, Thompson CB. Akt-directed glucose metabolism can prevent Bax conformation change and promote growth factorindependent survival. Mol Cell Biol 2003; 23: 7315-7328.

10. Nutt LK, Margolis SS, Jensen M, Herman CE, Dunphy WG, Rathmell JC et al. Metabolic regulation of oocyte cell death through the CaMKII-mediated phosphorylation of caspase-2. Cell 2005; 123: 89-103.

11. Sui D, Wilson JE. Structural determinants for the intracellular localization of the isozymes of mammalian hexokinase: intracellular localization of fusion constructs incorporating structural elements from the hexokinase isozymes and the green fluorescent protein. Arch Biochem Biophys 1997; 345: 111-125.

12. Vallabhapurapu S, Karin M. Regulation and function of NF-kappaB transcription factors in the immune system. Ann Rev Immunol 2009; 27: 693-733.

13. Voll RE, Jimi E, Phillips RJ, Barber DF, Rincon M, Hayday AC et al. NF-kappa B activation by the pre-T cell receptor serves as a selective survival signal in $T$ lymphocyte development. Immunity 2000; 13: 677-689.

14. Yang B, Hodgkinson A, Oates PJ, Millward BA, Demaine AG. High glucose induction of DNA-binding activity of the transcription factor NFkappaB in patients with diabetic nephropathy. Biochimica et Biophysica Acta 2008; 1782: 295-302.

15. Li H, Telemaque S, Miller RE, Marsh JD. High glucose inhibits apoptosis induced by serum deprivation in vascular smooth muscle cells via upregulation of Bcl-2 and Bcl-xl. Diabetes 2005; 54: 540-545.

16. James LR, Tang D, Ingram A, Ly H, Thai $K$, Cai $L$ et al. Flux through the hexosamine pathway is a determinant of nuclear factor kappaB- dependent promoter activation. Diabetes 2002; 51: 1146-1156.

17. Iwasaki Y, Kambayashi M, Asai M, Yoshida M, Nigawara T, Hashimoto K. High glucose alone, as well as in combination with proinflammatory cytokines, stimulates nuclear factor kappa-B-mediated transcription in hepatocytes in vitro. J Diabetes Complications 2007; 21: 56-62.

18. Humphrey BD, Rudrappa SG. Increased glucose availability activates chicken thymocyte metabolism and survival. J Nutr 2008; 138: 1153-1157.

19. Gorer PA. Studies in antibody response of mice to tumour inoculation. Br J Cancer 1950; 4 : 372-379. 
20. Conte D, Liston $\mathrm{P}$, Wong JW, Wright KE, Korneluk RG. Thymocyte-targeted overexpression of xiap transgene disrupts $\mathrm{T}$ lymphoid apoptosis and maturation. Proc Natl Acad Sci USA 2001; 98: 5049-5054.

21. Bouillet $\mathrm{P}$, Purton JF, Godfrey DI, Zhang LC, Coultas L, Puthalakath $\mathrm{H}$ et al. BH3-only Bcl-2 family member Bim is required for apoptosis of autoreactive thymocytes. Nature 2002; 415 922-926.

22. Khaled AR, Li WQ, Huang J, Fry TJ, Khaled AS, Mackall CL et al. Bax deficiency partially corrects interleukin-7 receptor alpha deficiency. Immunity 2002; 17: 561-573.

23. Deveraux QL, Leo E, Stennicke HR, Welsh K, Salvesen GS, Reed JC. Cleavage of human inhibitor of apoptosis protein XIAP results in fragments with distinct specificities for caspases. EMBO J 1999; 18: 5242-5251.

24. Kucharczak J, Simmons MJ, Fan Y, Gelinas C. To be, or not to be: NF-kappaB is the answer - role of Rel/NF-kappaB in the regulation of apoptosis. Oncogene 2003; 22 : 8961-8982.

25. Prendes $M$, Zheng $Y$, Beg AA. Regulation of developing $B$ cell survival by RelA-containing NF-kappa B complexes. J Immunol 2003; 171: 3963-3969.

26. Munzert G, Kirchner D, Stobbe H, Bergmann L, Schmid RM, Dohner H et al. Tumor necrosis factor receptor-associated factor 1 gene overexpression in B-cell chronic lymphocytic leukemia: analysis of NF-kappa B/Rel-regulated inhibitors of apoptosis. Blood 2002; 100: 3749-3756.

27. Alcamo E, Mizgerd JP, Horwitz BH, Bronson R, Beg AA, Scott M et al. Targeted mutation of TNF receptor I rescues the RelA-deficient mouse and reveals a critical role for NF-kappa B in leukocyte recruitment. J Immunol 2001; 167: 1592-1600.

28. Liou HC, Jin Z, Tumang J, Andjelic S, Smith KA, Liou ML. c-Rel is crucial for lymphocyte proliferation but dispensable for T cell effector function. Int Immunol 1999; 11: 361-371.

29. Yao D, Brownlee M. Hyperglycemia-induced reactive oxygen species increase expression of the receptor for advanced glycation end products (RAGE) and RAGE ligands. Diabetes 2010; 59: 249-255.
30. Chen J, Song M, Yu S, Gao P, Yu Y, Wang H et al. Advanced glycation endproducts alter functions and promote apoptosis in endothelial progenitor cells through receptor for advanced glycation endproducts mediate overpression of cell oxidant stress. $\mathrm{Mol}$ Cell Biochem 2010; 335: 137-146.

31. Devaraj S, Jialal I. Increased secretion of IP-10 from monocytes under hyperglycemia is via the TLR2 and TLR4 pathway. Cytokine 2009; 47: 6-10.

32. Irla $\mathrm{M}$, Hollander $\mathrm{G}$, Reith $\mathrm{W}$. Control of central self-tolerance induction by autoreactive CD4+ thymocytes. Trends Immunol 2010; 31: 71-79.

33. Bonasio R, Scimone ML, Schaerli P, Grabie N, Lichtman AH, von Andrian UH. Clonal deletion of thymocytes by circulating dendritic cells homing to the thymus. Nat Immunol 2006; 7: 1092-1100.

34. Zucchelli S, Holler P, Yamagata T, Roy M, Benoist C, Mathis D. Defective central tolerance induction in NOD mice: genomics and genetics. Immunity 2005; 22: 385-396.

35. Linette GP, Grusby MJ, Hedrick SM, Hansen TH, Glimcher LH, Korsmeyer SJ. Bcl-2 is upregulated at the $\mathrm{CD} 4+\mathrm{CD} 8+$ stage during positive selection and promotes thymocyte differentiation at several control points. Immunity 1994; 1: 197-205.

36. Yang WH, Park SY, Nam HW, Kim do H, Kang JG, Kang ES et al. NFkappaB activation is associated with its O-GlcNAcylation state under hyperglycemic conditions. Proc Natl Acad Sci USA 2008; 105: 17345-17350.

37. Kane LP, Shapiro VS, Stokoe D, Weiss A. Induction of NF-kappaB by the Akt/PKB kinase. Curr Biol 1999; 9: 601-604.

38. Hajduch E, Litherland GJ, Hundal HS. Protein kinase B (PKB/Akt) - a key regulator of glucose transport? FEBS Lett 2001; 492: 199-203.

39. Maedler K, Spinas GA, Lehmann R, Sergeev P, Weber M, Fontana A et al. Glucose induces beta-cell apoptosis via upregulation of the Fas receptor in human islets. Diabetes 2001; 50: 1683-1690.

40. Wu KK, Huan Y. Streptozotocin-induced diabetic models in mice and rats. Curr Protocols Pharmacol 2008; 40: 5.47.1-5.47.14

Supplementary Information accompanies the paper on Cell Death and Differentiation website (http://www.nature.com/cdd) 